\title{
Analysis of Aurora B kinase in non-Hodgkin lymphoma
}

\author{
Takayuki Ikezoe ${ }^{1}$, Tamotsu Takeuchi ${ }^{2}$, Jing Yang ${ }^{1}$, Yoshihiro Adachi ${ }^{2}$, Chie Nishioka ${ }^{1}$, Mutsuo Furihata ${ }^{2}$, \\ $\mathrm{H}$ Phillip Koeffler ${ }^{3}$ and Akihito Yokoyama ${ }^{1}$
}

This study explored the levels of Aurora B, a key regulator of mitosis, in 71 lymph nodes and tumor specimens excised operatively from individuals with various types of non-Hodgkin lymphoma (NHLs). Immunohistochemical examination found that diffuse large B-cell lymphoma $(10 / 21,48 \%)$ and Burkitt lymphoma (BL) $(6 / 7,86 \%)$ cells highly (percentage of positive cells, $>20 \%$ ) expressed Aurora B in their nuclei. On the other hand, none of the low-grade B-cell lymphoma $(n=20)$, except for one case of follicular lymphoma, highly expressed this protein kinase, suggesting that levels of Aurora B correlated with histological grade in B-cell NHLs $(P<0.01)$. Exposure of BL/leukemia cells to AZD1152-HQPA in vitro, a selective inhibitor of Aurora B kinase, potently induced growth arrest and apoptosis in a caspase-dependent, as well as -independent manner. Moreover, AZD1152 synergistically enhanced the effects of vincristine (VCR) to induce growth arrest of these cells. Further experiments found that VCR increased levels of the p-Aurora B through the activation of c-Jun N-terminal kinase, which was blocked in the presence of AZD1152-HQPA. Laboratory Investigation (2009) 89, 1364-1373; doi:10.1038/labinvest.2009.106; published online 12 October 2009

KEYWORDS: aurora B; burkitt lymphoma; AZD1152; apoptosis

The Aurora family of serine/threonine kinases has an important role in chromosome alignment, segregation and cytokinesis during mitosis. The Aurora family consists of three members: Aurora A, B and C, which share 67-76\% amino acid sequence identity in their catalytic domains, and few similarities in their N-terminus. ${ }^{1,2}$ Aurora A localizes on centrosomes and has a crucial role in each step of mitosis including bipolar spindle formation. ${ }^{2}$ Aurora B is a chromosomal passenger protein and localizes at centromeres during prometaphase and subsequently relocates to midzone microtubules and midbodies during anaphase and telophase. $^{1,3}$ Aurora B has a role in chromosome alignment, kinetochore-microtubule biorientation, activation of the spindle assembly checkpoint and cytokinesis in association with phosphorylation of histone $\mathrm{H} 3$ on Ser $10 .{ }^{1,3}$ Aurora $\mathrm{C}$ is specifically expressed in the testis and has a role in spermatogenesis. ${ }^{4}$ Recent studies found that Aurora $\mathrm{C}$ also acted as a chromosomal passenger protein and might compensate for Aurora B function. ${ }^{5}$

Several studies found that Aurora A and B were overexpressed in a variety of solid tumors, including colon, ${ }^{6,7}$ breast, ${ }^{7-10}$ prostate, ${ }^{11}$ pancreas, ${ }^{12}$ thyroid, ${ }^{13}$ and head and neck cancers. ${ }^{14}$ We have shown recently that hematological malignant cells including those from acute myeloid leukemia (AML), acute lymphoblastic leukemia (ALL), as well as chronic myeloid leukemia aberrantly expressed Aurora A and B kinases. ${ }^{15}$ In addition, we have shown that ZM447439, a novel and selective inhibitor of Aurora A and B kinases, effectively induced growth arrest and apoptosis of a variety of types of leukemia cells. ${ }^{15}$ Studies conducted by other groups noted that the phenotype of HeLa cells after their exposure to ZM447439 was similar to that induced by knock down of the Aurora B kinase gene. ${ }^{16} \mathrm{We}$, therefore, hypothesized that Aurora B might be a more favorable molecular target for cancer therapy than Aurora A.

AZD1152 is a novel acetanilide-substituted pyrazole-aminoquinazoline prodrug that is converted rapidly to the active drug, AZD1152 hydroxy-QPA (AZD1152-HQPA), in human plasma. ${ }^{17}$ AZD1152-HQPA is a specific inhibitor of the enzymatic activity of Aurora kinase, with selectivity for Aurora B (IC50 of $0.36 \mathrm{nM}$ versus $1369 \mathrm{nM}$ for Aurora B and A kinases, respectively); the inhibitor had less activity against a panel of more than 50-fold against other serine-threonine and tyrosine kinases including FLT3, JAK2 and Abl. ${ }^{17,18}$ AZD1152 was active against a variety of solid tumors as well as hematological malignant cells. ${ }^{18,19}$

\footnotetext{
${ }^{1}$ Department of Hematology and Respiratory Medicine, Kochi University, Kochi, Japan; ${ }^{2}$ Department of Pathology, Kochi University, Kochi, Japan; ${ }^{3}$ Cedars-Sinai Medical Center, UCLA School of Medicine, Los Angeles, CA, USA

Correspondence: Dr T Ikezoe, MD, Department of Hematology and Respiratory Medicine, Kochi University, Okoh-cho, Nankoku, Kochi, 783-8505, Japan.

E-mail: ikezoet@kochi-u.ac.jp

Received 19 June 2009; revised 11 August 2009; accepted 17 August 2009
} 
c-Jun NH2-terminal kinase (JNK; also known as stressactivated protein kinase) is one of the mitogen-activated protein kinase superfamily. ${ }^{20} \mathrm{JNK}$ is known to be activated in response to certain growth factors, as well as environmental stresses such as ultraviolet radiation. Activated JNK mediates both apoptotic and prosurvival signal pathways. For example, 12-O-tetradecanoyl phorbol 13-acetate-induced phosphorylation of JNK, resulting in apoptosis of androgen-dependent human prostate cancer cells. ${ }^{21}$ On the other hand, activation of JNK was required for interleukin-3-dependent proliferation of $\mathrm{Ba} / \mathrm{F} 3$ murine pre- $\mathrm{B}$ cells. ${ }^{22}$ In addition, JNK was shown to be required for survival and proliferation of B-lymphoma cells. ${ }^{23}$

This study analyzed the expression of Aurora B in various types of non-Hodgkin lymphoma (NHLs) by immunohistochemistry and found that this kinase was highly expressed in histologically aggressive types of lymphoma such as diffuse large B cell (DLBC) and Burkitt lymphoma (BL). Inhibition of Aurora B by AZD1152 caused apoptosis of these cells in parallel with activation of the caspase pathway. In addition, AZD1152 synergistically enhanced the ability of vincristine (VCR), a tubulin-depolymerizing agent, to induce apoptosis of BL cells. Moreover, we found that VCR induced activation of Aurora kinases through stimulation of JNK, which was blocked by AZD1152 in BL cells.

\section{MATERIALS AND METHODS} Immunohistochemistry

A total of 71 paraffin-embedded malignant lymphoma samples were obtained by the Laboratory of Diagnostic Pathology of Kochi University Hospital during the period from 1992 to 2008. All specimens were obtained at the initial presentation of the patients. Patients' ages ranged from 23 to 95 years with a mean age of 65 years. Histology of malignant lymphoma was diagnosed according to the WHO classification by at least two experienced pathologists (TT and MF). This study included DLBC lymphoma (DLBCL, 21 cases), BL (7 cases), follicular lymphoma (FL, 13 cases), mantle cell lymphoma (MCL, 4 cases), mucosa-associated lymphoid tissue lymphoma (MALT, 3 cases), adult T-cell leukemia/ lymphoma (11 cases), peripheral T-cell lymphoma (9 cases), and Hodgkin lymphoma (3 cases). In total, 3 (two DLBCL and one MCL) out of 48 patients with B-cell lymphoma were diagnosed before 2001, and were treated with CHOP (cyclophosphamide, adriamycin, VCR and prednisone). Rest of the patients were treated with retuximab in combination with anti-cancer agents. The immunohistochemical procedure was performed with a Ventana DISCOVERY autostainer system according to the protocol provided by the manufacturer (Ventana Japan, Osaka, Japan). The anti-Aurora B antibody (EP1009Y, Epitomics, Burlingame, CA, USA) was used. The tissue sections were counterstained with hematoxylin and semiquantitatively scored as weakly positive $(+;>5$ to $20 \%)$, moderately positive $(++;>20$ to $80 \%)$ or markedly positive $(+++;>80 \%)$.
The study was approved by the Ethics Committee of Kochi University (approved \#20-54), and complied with the Helsinki Declaration.

\section{Cells}

Burkitt lymphoma/leukemia cell lines, BALM14, -18 and -27 were kind gifts from Dr A Hrashima (Hayashibara Biochemical Laboratories, Okayama, Japan). Daudi and Ramos Burkitt lymphoma cell lines were obtained from RIKEN BRC Cell Bank (Tsukuba, Japan). Acute promyelocytic leukemia NB4 and AML MOLM-13 cell lines were a kind gift from $\operatorname{Dr} M$ Lanotte (St Louis Hospital, Paris, France) and Dr Y Matsuo (Fujisaki Cell Center, Hayashibara Biochemical Laboratories), respectively. Bone marrow mononuclear cells and peripheral blood lymphocytes were isolated from healthy volunteers after obtaining informed consent, as previously described. ${ }^{24}$

\section{Reagents}

AZD1152-HQPA was provided by AstraZeneca (Macclesfield, UK). AZD1152-HQPA was dissolved in 100\% dimethyl sulfoxide (DMSO; Burdick \& Jackson, Muskegon, MI, USA) to a stock concentration of $10 \mathrm{mM}$ and stored at $-80^{\circ} \mathrm{C}$. The pan-caspase inhibitor $\mathrm{z}$-Val-Ala-Asp (OMe)-Fluoromethylketone (z-VAD-FMK) and the JNK inhibitor SP600125 were purchased from BIOMOL International (Plymouth Meeting, PA, USA) and Calbiochem (San Diego, CA, USA), respectively, and were dissolved in $100 \%$ DMSO to a stock concentration of $20 \mathrm{mM}$.

\section{3-(4,5-dimethylthiazol-2-yl)-2,5-Diphenyltetrazolium Bromide (MTT) Assays}

Cells $\left(5 \times 10^{5}\right.$ per $\left.\mathrm{ml}\right)$ were cultured with various concentrations of AZD1152-HQPA for 2 days in 96-well plates. After culture, MTT assay was performed as previously described. ${ }^{25}$ All experiments were performed in triplicate and repeated at least thrice.

\section{Measurement of p-Histone $\mathrm{H} 3$ at Ser10 and P-JNK by Flow Cytometry}

The Alexa Fluor 488-conjugated anti-p-histone H3 (Ser10, Cell Signaling Technology, Beverly, MA, USA) and antip-JNK (SC-6254, Santa Cruz, Santa Cruz, CA, USA) antibodies were used to quantify the population of leukemia/ lymphoma cells expressing the phosphorylated forms of histone $\mathrm{H} 3$ and JNK, respectively. These experiments were performed using flow cytometry.

\section{Immunoblotting}

Immunoblotting was performed as previously described. ${ }^{26}$ The antibodies used were anti-PARP (Cell Signaling Technology), anti-caspase 3 (Cell Signaling Technology), antiGAPDH (Abcam Japan, Tokyo, Japan), anti-Aurora B kinase (Cell Signaling Technology) and anti-p-Aurora A/B/C kinases (Cell Signaling Technology). Band intensities were measured by densitometry. 


\section{Small interfering RNA}

Control siRNA and a siRNA against JNK were purchased from Sigma (St Louis, MO, USA). Control siRNA targets green fluorescence proteins.

\section{Transfections}

NB4 cells were transiently transfected with either control or JNK siRNA by Amaxa electroporator Nucleofector II (Wako Pure Chemical Industries, Osaka, Japan), using the Nucleofector Kit V (program X-001). The preliminary experiments using the green fluorescence protein-expressing vector found that efficacy of transfection with this program was approximately $70 \%$, with approximately $80 \%$ cell viability, as measured by FACS and annexin V staining (data not shown). We used NB4 cells for these studies, because none of BL/leukemia cell lines were suitable for transfection (data not shown). We previously showed that NB4 cells expressed Aurora kinases and the inhibitor of these kinases effectively inhibited their proliferation. ${ }^{15}$
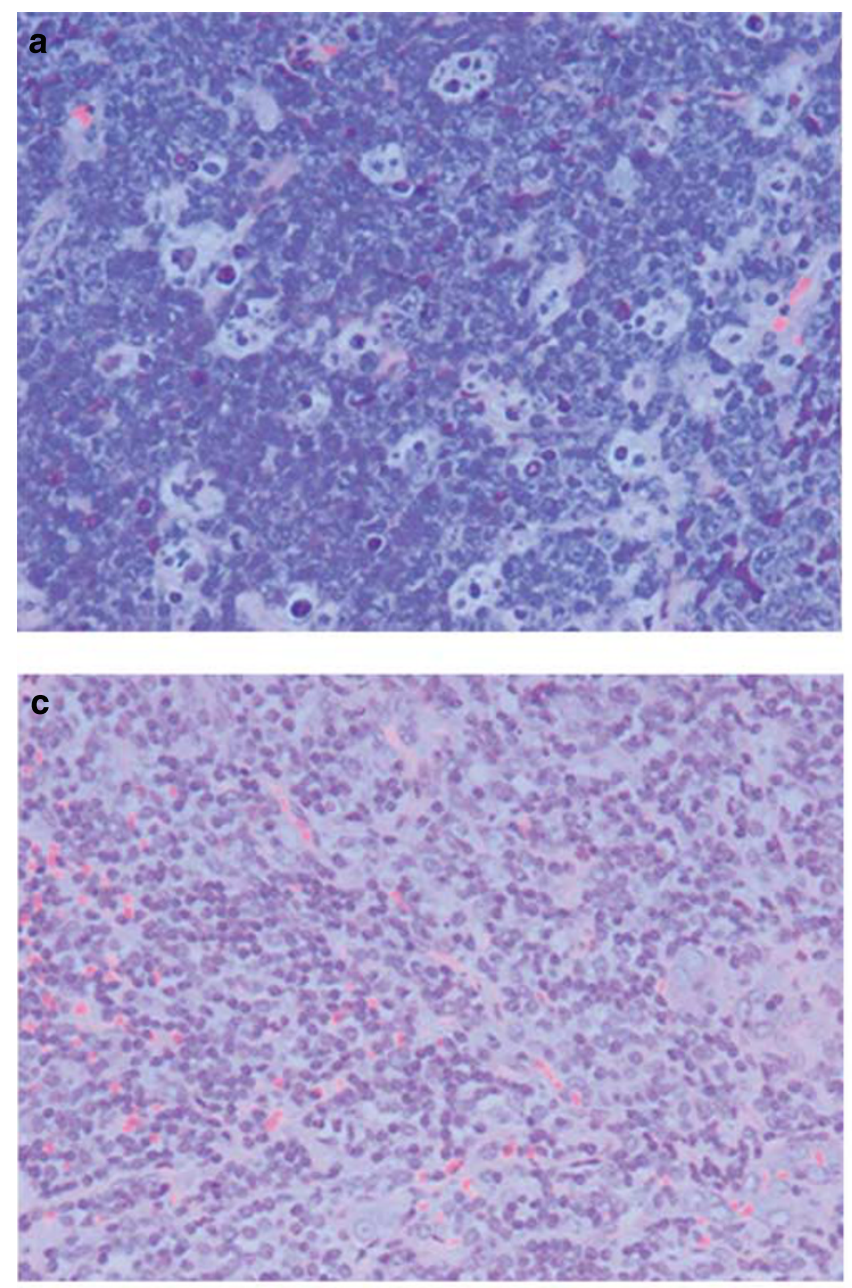

\section{Data analysis}

The $\chi^{2}$-test was used to examine the relation between levels of Aurora B and clinical factors. The combination index (CI) for growth inhibition elicited by AZD1152-HPQA and VCR in leukemia cells was calculated using the median effect method of Chou and Talalay ${ }^{27}$ (Calcusyn Software available from Biosoft, Cambridge, United Kingdom). CI values $<1$ indicate synergy, a $\mathrm{CI}=1$ shows an additive effect and a $\mathrm{CI}$ $>1$ shows antagonism between the two agents.

\section{RESULTS \\ High-Grade B-cell Lymphoma Cells Aberrantly Expressed Aurora B}

Representative results of immunohistochemistry exploring the expression of Aurora $\mathrm{B}$ in $\mathrm{BL}$ and $\mathrm{FL}$ were shown in Figure 1. DLBCL $(10 / 21,48 \%)$ and BL $(6 / 7,86 \%)$ samples highly expressed Aurora B in their nuclei (percentage of positive cells, $>20 \%$ ) (Table 1). On the other hand, FL $(12 / 13,92 \%)$, MCL $(1 / 4,25 \%)$ and MALT $(1 / 3,33 \%)$ samples slightly expressed Aurora B (percentage of positive cells,
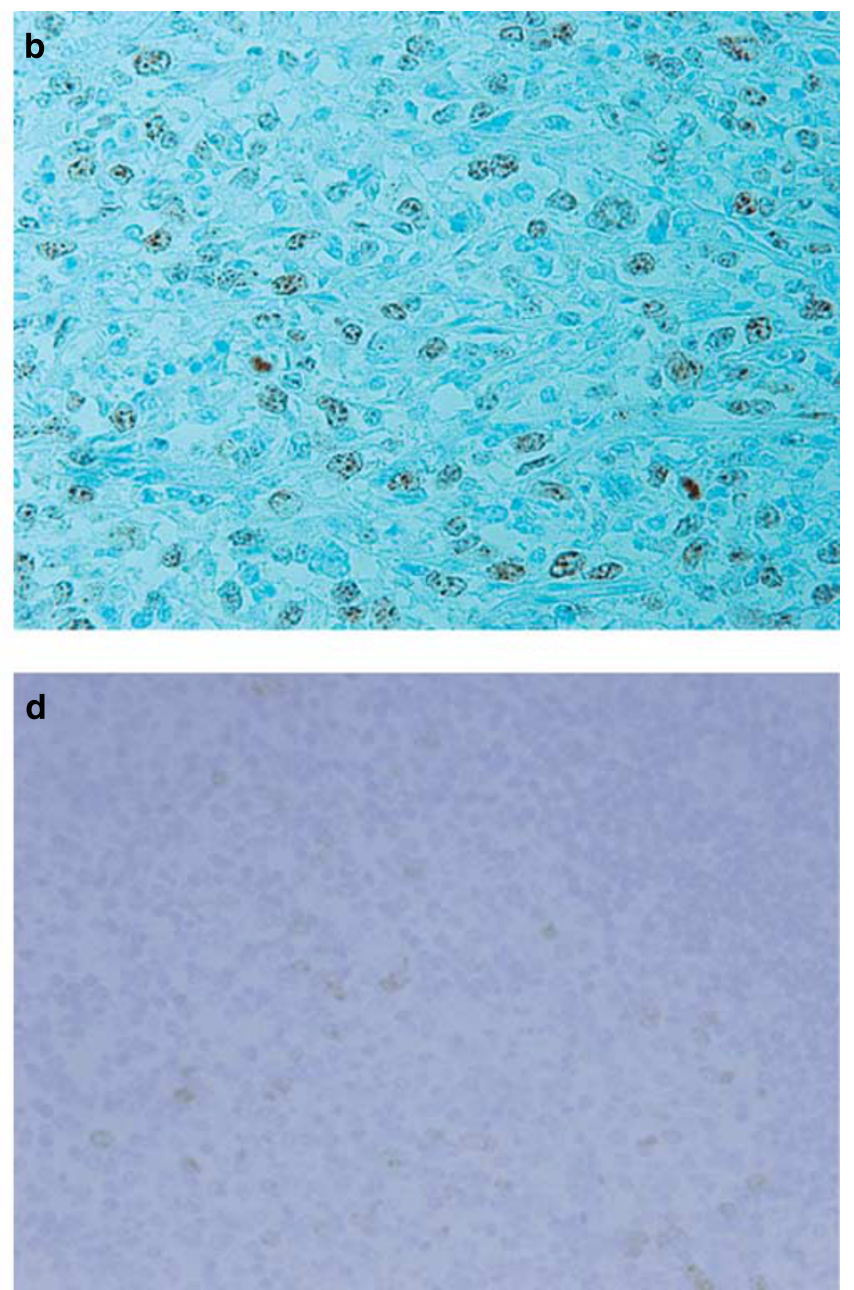

Figure 1 Representative histology and immunohistochemistry of lymph nodes from individuals with Burkitt lymphoma (BL) and follicular lymphoma (FL). Hematoxylin and eosin staining of BL (a) and FL (c). Lymph nodes from BL (b) and FL (d) were stained with anti-Aurora B antibody. Original magnifications were $\times 400$. 
Table 1 Expression of Aurora B in malignant lymphomas

\begin{tabular}{llllll}
\hline Levels of Aurora B & - & + & + & ++ & ++ \\
& No. (\%) & No. (\%) & No. (\%) & No. (\%) & Total \\
\hline
\end{tabular}

$\begin{array}{lrlrlll}\begin{array}{l}\text { Age at diagnosis } \\ <65 \text { years }\end{array} & 9(28) & 17(53) & 6(19) & 0(0) & 32 & P=0.018 \\ >65 \text { years } & 11(28) & 12(31) & 11(28) & 5(13) & 39\end{array}$

$\begin{array}{lrrrrrrr}\text { Gender } & & & & & & P>0.05 \\ \text { Male } & 8(24) & 16(48) & 7(21) & 2(6) & 33 & \\ \text { Female } & 11(29) & 14(37) & 10(26) & 3(8) & 38 & \end{array}$

$\begin{array}{lllllrl}\text { Histology } & & & & & P<0.01 \\ \text { DLBCL } & 2(10) & 9(43) & 9(43) & 1(5) & 21 & \\ \text { BL } & 0(0) & 1(14) & 3(43) & 3(43) & 7 & 13 \\ \text { FL } & 0(0) & 12(92) & 1(8) & 0(0) & 13 \\ \text { MCL } & 3(75) & 1(25) & 0(0) & 0(0) & 4 \\ \text { MALT } & 2(67) & 1(33) & 0(0) & 0(0) & 3 \\ \text { HL } & 1(33) & 2(67) & 0(0) & 0(0) & 3 \\ \text { ATL } & 8(73) & 0(0) & 3(27) & 0(0) & 11 \\ \text { PTCL } & 3(33) & 5(56) & 0(0) & 1(11) & 9\end{array}$

\begin{tabular}{lrrrllll} 
Clinical stage & & & & & & $P=0.024$ \\
I/II & $13(38)$ & $13(38)$ & $8(24)$ & $0(0)$ & 34 & \\
III/IV & $6(16)$ & $18(49)$ & $8(22)$ & $5(14)$ & 37 & \\
& & & & & & \\
IPI & & & & & & $P<0.01$ \\
Low/low-int & $16(31)$ & $25(49)$ & $10(20)$ & $0(0)$ & 51 & \\
High/high-int & $4(20)$ & $4(20)$ & $7(35)$ & $5(25)$ & 20 & \\
\hline
\end{tabular}

ATL, adult T-cell lymphoma; BL, Burkitt lymphoma; DLBCL, diffuse large B-cell lymphoma; FL, follicular lymphoma; HL, Hodgkin lymphoma; IPI, International Prognostic Index; int, intermediate; MCL, mantle cell lymphoma; MALT, mucosa-associated lymphoid tissue lymphoma; PTCL, peripheral T-cell lymphoma. Statistical significance of difference between age $<65$ years and $>65$ years, male and female, DLBCL+BL and FL+MCL+MALT, clinical stage I/II and III/IV, and IPI low/low-int and high/high-int was determined by $\chi^{2}$-test.

5-20\%) (Figure 1b) and none of them, except for one case of FL, highly expressed this protein kinase, suggesting that expression of Aurora B correlated with histological grade in B-cell NHLs $(P<0.01)$. In addition, levels of Aurora B correlated with advanced clinical stage $(P=0.024)$ and high International Prognostic Index (IPI) score $(P<0.01)$ (Table 1$)$.

\section{Inhibition of Aurora B by AZD1152-HQPA Induced Growth Arrest and Apoptosis of BL/Leukemia Cells}

We examined whether Aurora B could be a molecular target for the treatment of NHLs using BL/leukemia cells. Ramos, Daudi, BALM-14, -18 and -27 BL/leukemia cells expressed
Aurora B protein as measured by western blot analysis (Figure 2a). On the other hand, bone marrow mononuclear cells as well as peripheral blood lymphocytes from healthy volunteers did not express this kinase protein (Figure 2a). Ph-positive B-ALL PALL-2 and AML MOLM-13 cells were used as a positive control. Exposure of BL/leukemia cells to various concentrations of AZD1152-HQPA (12.5-100 nM) for 2 days effectively inhibited their proliferation with IC50s between 50 and $100 \mathrm{nM}$, as measured by MTT assay (Figure $2 \mathrm{~b})$. As expected, AZD1152-HQPA (12.5-100 nM) did not affect the viability of peripheral blood lymphocytes from healthy volunteers (Figure $2 b$ ).

Western blot analysis found that exposure of Ramos, BALM-14 and -27 cells to AZD1152-HQPA (12.5-100 nM) for 2 days increased levels of the cleaved forms of PARP and caspase 3, suggesting that AZD1152-HQPA induced apoptosis of these cells (Figure 2c). Cleavage of PARP in Daudi cells was not associated with activation of caspase 3 (Figure 2c). Other caspases such as caspase 7 or 2 could cleave PARP, leading to the apotosis of Daudi cells. Pre-incubation of Ramos and BALM-27 cells with the pan-caspase inhibitor Z-VAD-FMS $(50 \mu \mathrm{M}, 1 \mathrm{~h})$ blocked AZD1152-HQPA-induced cleavage of PARP and caspase 3; and AZD1152-HQPAmediated growth inhibition of these cells was blunted (Figure 2d). These observations suggested that AZD1152-HQPA-induced growth inhibition of BL/leukemia cells was, at least in part, caspase dependent.

\section{AZD1152-HQPA Potentiated the Antiproliferative Activity of VCR Directed Against BALM-27 cells}

We examined the ability of AZD1152-HQPA to enhance the antiproliferative effects of the conventional anti-lymphoma/ leukemia agent, VCR. BALM-27 cells were cultured with various concentrations of AZD1152-HQPA (15-100 nM) and/or VCR $(0.625-5 \mathrm{nM})$ for $48 \mathrm{~h}$; and proliferation was measured by MTT assay (Figure 3a). AZD1152-HQPA $(30 \mathrm{nM})$ or VCR $(2.5 \mathrm{nM})$ alone inhibited MTT activity by approximately 25 and $30 \%$, respectively (Figure $3 \mathrm{a}$ ). When cells were exposed to both compounds in combination, proliferation was inhibited by approximately $80 \%$ (Figure 3a), resulting in a CI value of $<1$ (synergistic antiproliferative effect, figure not shown).

\section{VCR Activated Aurora Kinases}

We next attempted to verify the molecular mechanisms by which inhibition of Aurora B potentiated the effects of VCR in BALM-27 cells. Exposure of BALM-27 cells to various concentrations of VCR $(1.25-5 \mathrm{nM}, 24 \mathrm{~h})$ induced phosphorylation of Aurora kinase and its substrate p-Histone H3 in a dose-dependent manner, which were effectively blocked in the presence of AZD1152-HQPA ( $25 \mathrm{nM})$, as measured by FACS (Figures 3b,c). Western blot analysis confirmed that VCR $(1.25-5 \mathrm{nM}, 24 \mathrm{~h})$ stimulated the phosphorylation of Aurora kinases A and B in a dose-dependent manner, and 
a

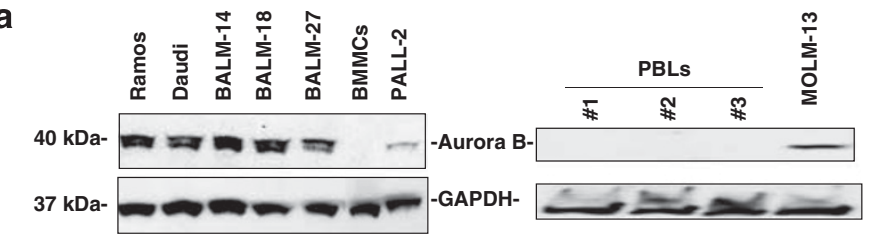

C
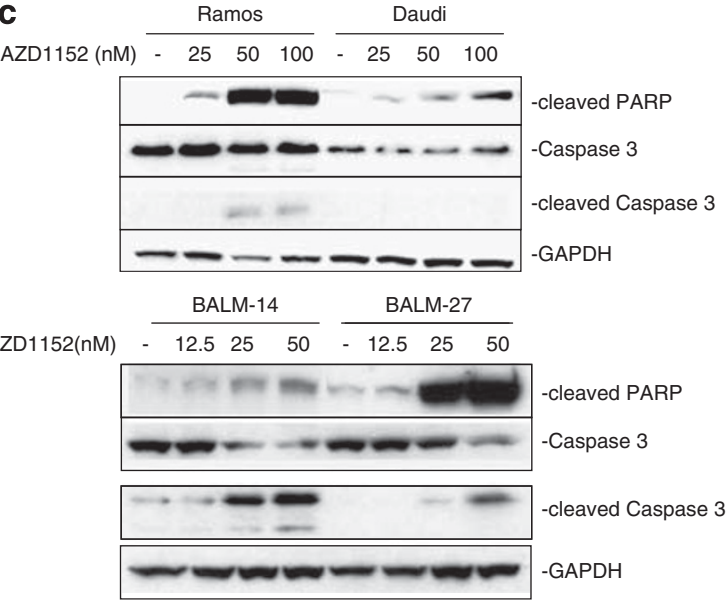
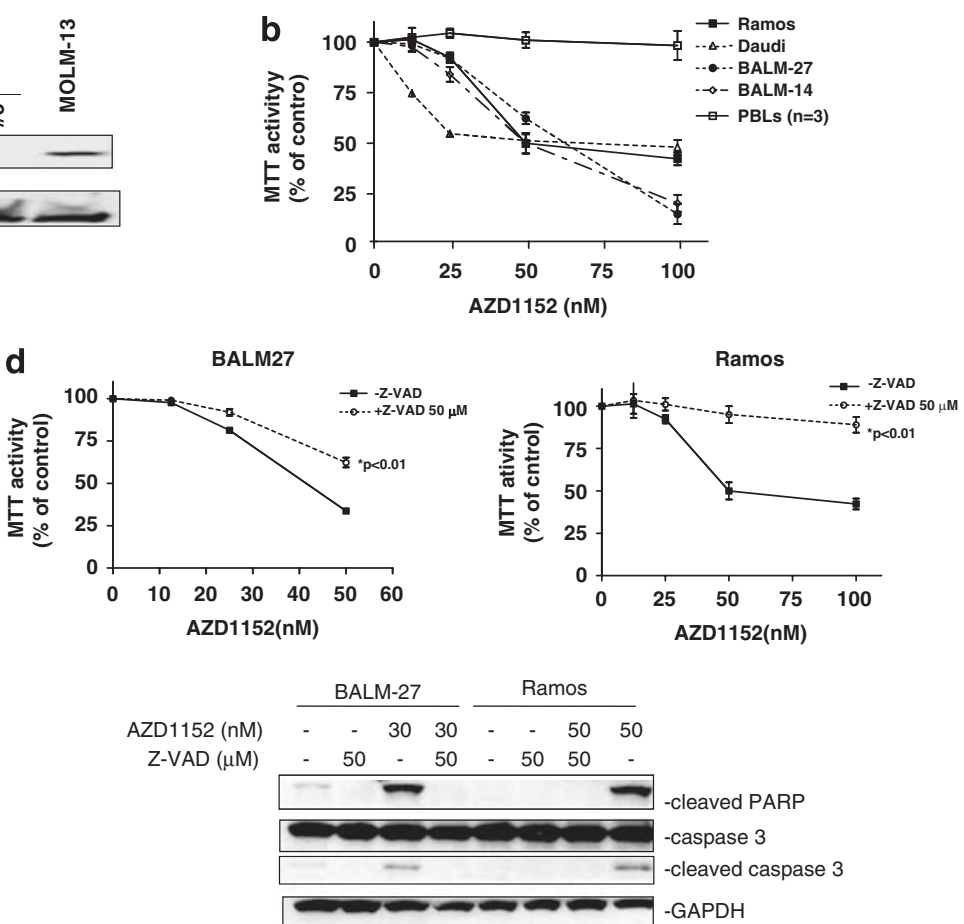

Figure 2 Burkitt lymphoma/leukemia cells express Aurora B. (a) Western blot analysis. Cell lysates from Burkitt lymphoma/leukemia cells and bone marrow mononuclear cells and peripheral blood lymphocytes from healthy volunteers were prepared and subjected to western blot analysis. The membrane was sequentially probed with anti-Aurora B and anti-GAPDH antibodies. PALL-2 and MOLM-13 cells were used as a positive control. (b) MTT assay. Burkitt lymphoma/leukemia and PBLs cells from healthy volunteers $(n=3)$ were exposed to various concentrations of AZD1152 $(12.5-100 \mathrm{nM})$. After $48 \mathrm{~h}$, cell viability was measured by MTT assay. Results represent the mean $\pm \mathrm{s} . \mathrm{d}$. of three experiments performed in triplicate. (c) AZD1152-HQPA induces apoptosis of Burkitt lymphoma/leukemia cells. Western blot analysis. Cells were exposed to various concentrations of AZD1152 $(12.5-100 \mathrm{nM})$. After $48 \mathrm{~h}$, cells were harvested, and proteins were extracted and subjected to western blot analysis. The membranes were sequentially probed with antibodies to cleaved PARP, cleaved caspase 3, caspase 3 and GAPDH. Results represent one of three experiments performed independently. (d) Pre-incubation of BALM-27 and Ramos cells with Z-VAD-FMS rescues cells from AZD1152-mediated growth inhibition and apoptosis. BALM-27 and Ramos cells were pre-incubated with either Z-VAD-FMS $(50 \mu \mathrm{M})(+\mathrm{ZAD})$ or control diluent $(-\mathrm{ZAD})$ for $1 \mathrm{~h}$ followed by the addition of AZD1152-HQPA (10-100 nM). After $48 \mathrm{~h}$, cell viability was measured by MTT assay. At the same time, cell lysates were prepared and subjected to western blot analysis. The membranes were sequentially probed with anti-cleaved PARP, -cleaved caspase 3, -caspase 3 and GAPDH antibodies. BMMCs, bone marrow mononuclear cells; GAPDH, glyceraldehyde 3-phosphate dehydrogenase; MTT, 3-(4,5-Dimethylthiazol-2-yl)-2,5-diphenyltetrazolium bromide; PARP, poly (ADP-ribose) polymerase; PBMCs, peripheral blood mononuclear cells; PBLs, peripheral blood lymphocytes.

AZD1152-HQPA (25 nM) effectively blocked p-Aurora B but not Aurora A (Figure 3d).

\section{JNK Mediated VCR-Stimulated Phosphorylation of Aurora Kinases}

Further experiments explored the signal pathways, which mediated VCR-induced phosphorylation of Aurora kinases. JNK was constitutively activated in BALM-27 cells as judged by the detection of p-JNK by FACS (Figure 4a). Exposure of
BALM-27 cells to VCR $(5 \mathrm{nM}, 1 \mathrm{~h})$ further stimulated the phosphorylation of JNK, which was effectively inhibited by the specific JNK inhibitor SP600125 $(5 \mu \mathrm{M})$ (Figure 4a). As expected, SP600125 (1.25-5 $\mu \mathrm{M})$ downregulated VCR ( $5 \mathrm{nM}$, $24 \mathrm{~h}$ ) induced expression of p-Aurora B in a dose-dependent manner (Figure $4 \mathrm{~b}$ ), suggesting that VCR induced p-Aurora B through JNK signaling. Surprisingly, SP600125 more profoundly blocked VCR-induced phosphorylation of Aurora A (Figure 4b).

Figure 3 AZD1152-HQPA potentiates effect of VCR. (a) MTT assay. BALM-27 cells were cultured with AZD1152-HQPA (15 or 30 nM) and/or vincristine (VCR) (0.625-5 nM). After 2 days, cell proliferation was measured by MTT assay. (b, c) VCR increases levels of p-Aurora A and B kinases and p-histone H3 (Ser10). FACS analysis. BALM-27 cells were exposed to VCR (1.25-5 nM) and/or AZD1152-HQPA (25 nM). After 3 h, p-Aurora A/B (b) and p-histone H3 (c) expressing populations were measured by FACScan. Results represent the mean \pm s.d. of the three experiments performed in duplicate plate. (d) Western blot analysis. BALM-27 cells were exposed to VCR (1.25-5 nM) and/or AZD1152-HQPA (3-30 nM). After $3 \mathrm{~h}$, cells were harvested, and proteins were extracted and subjected to western blot analysis. The membranes were sequentially probed with anti-p-Aurora $A / B / C$, anti-Aurora $A$ and $B$, and histone $H 1$ antibodies. Results represent one of the three experiments performed independently. FACS, fluorescence-activated cell sorting; MTT, 3-(4,5-Dimethylthiazol-2-yl)-2,5diphenyltetrazolium bromide. 


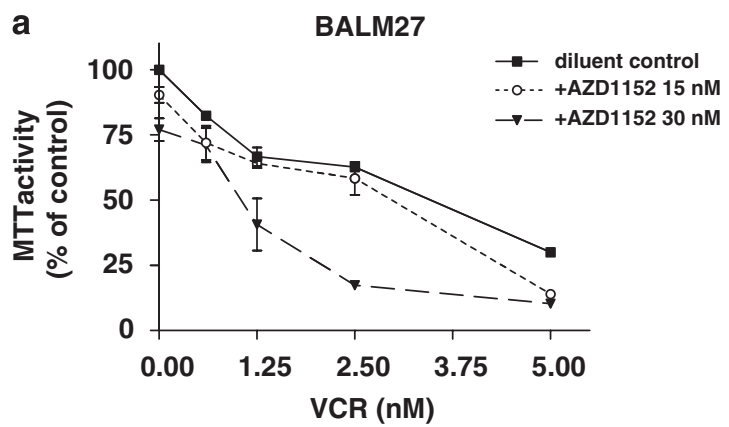

b

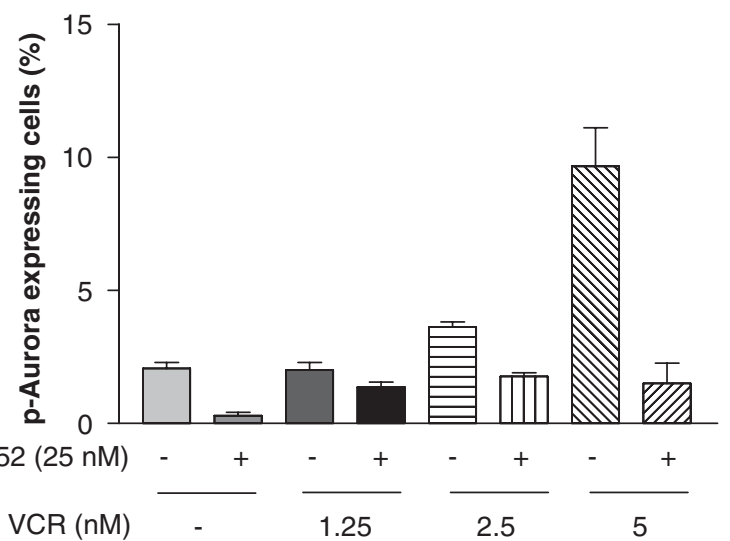

c

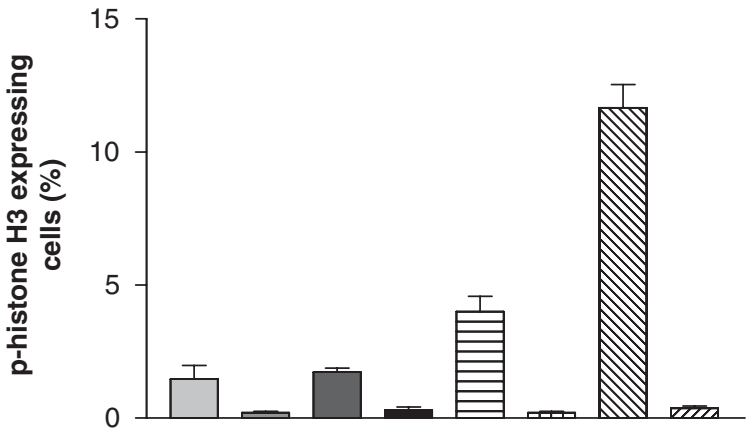

AZD1152 (25 nM)

$\operatorname{VCR}(\mathrm{nM})$

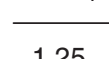

$\frac{+}{2.5} \frac{-+}{5}$

d
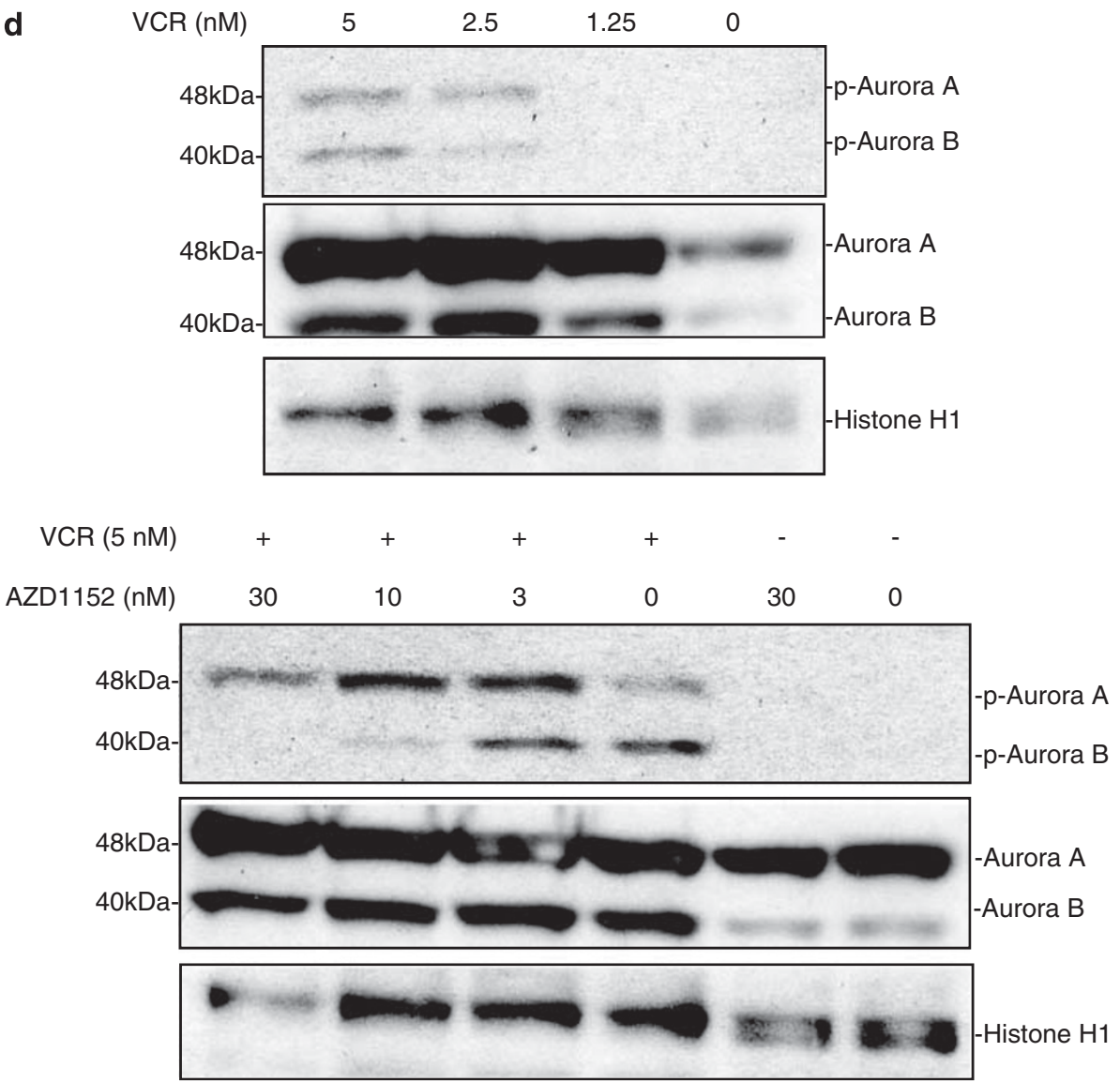

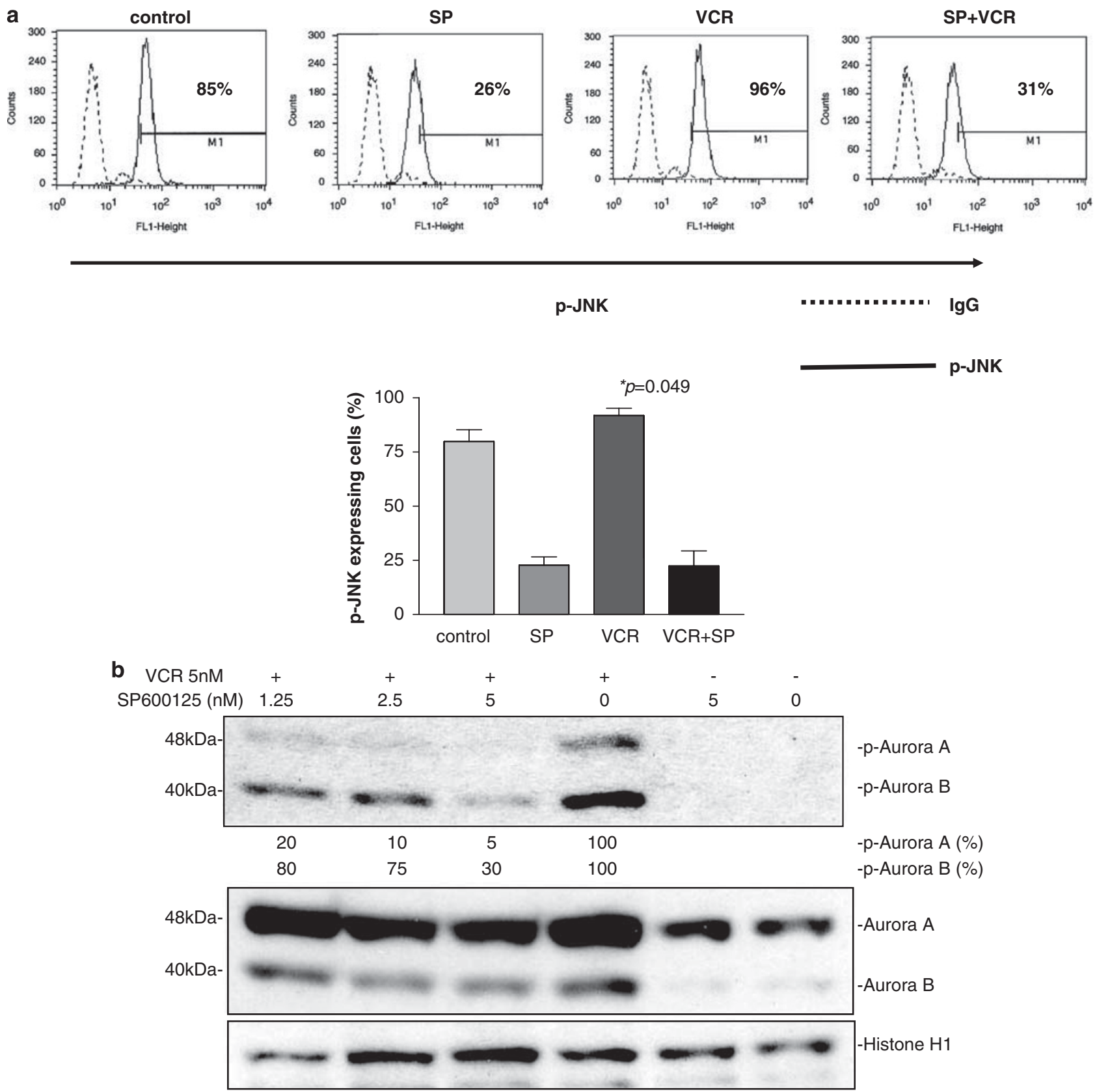

Figure 4 VCR activates JNK. (a) FACS analysis. BALM-27 cells were exposed to VCR ( $5 \mathrm{nM}$ ) and/or SP600125 (5 $\mu$ M). After $3 \mathrm{~h}$, p-JNK-expressing population was quantified by FACScan. The statistical significance of difference between control and VCR-treated cells was determined by Mann-Whitney U-test. The bar graph represents a mean \pm s.d. of three experiments performed in duplicate plate. (b) Western blot analysis. BALM- 27 cells were exposed to VCR (5 nM) and/or SP600125 (1.25-5 $\mu \mathrm{M})$. After $3 \mathrm{~h}$, cells were harvested, and proteins were extracted and subjected to western blot analysis. The membranes were sequentially probed with anti-p-Aurora $A / B / C$, anti-Aurora $A$ and $B$, and histone $\mathrm{H} 1$ antibodies. The band intensity was measured by densitometry. Results represent one of the three experiments performed independently. JNK, Jun N-terminal Kinase; SP, SP600125; VCR, vincristine.

\section{Downregulation of JNK by a siRNA Blunted VCR-Stimulated Activation of Aurora Kinase}

Moreover, we repressed JNK by a siRNA in NB4 leukemia cells. VCR $(0.03 \mathrm{nM}, 1 \mathrm{~h})$ stimulated activation of p-Aurora kinase was effectively inhibited in the JNK siRNA-transfected NB4 cells (Figure 5a). Notably, synergistic growth inhibition of NB4 cells mediated by combination of VCR and AZD1152 was significantly $(P<0.01)$ attenuated when JNK was downregulated in these cells (Figure $5 b$ ).

\section{Discussion}

This study, for the first time, showed that Aurora B was aberrantly expressed in histologically high-grade NHLs such as DLBCLs and BLs (Figure 1, Table 1). In addition, we found 
a

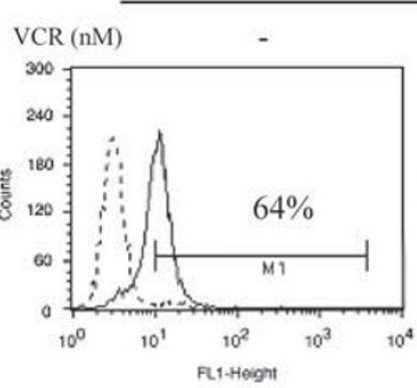

control siRNA

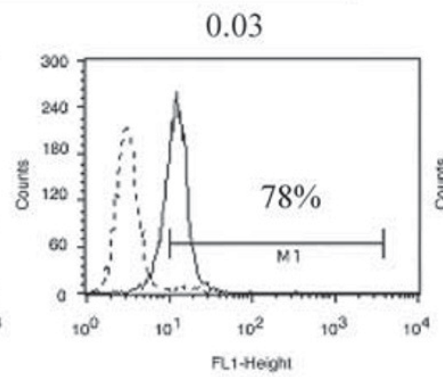

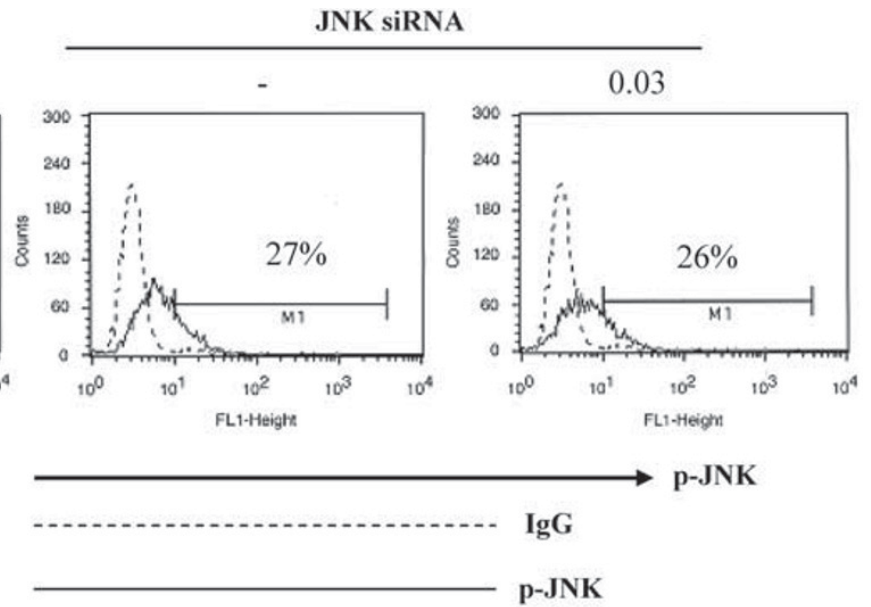

control siRNA

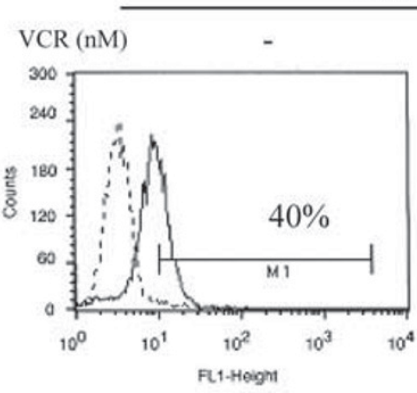

0.03
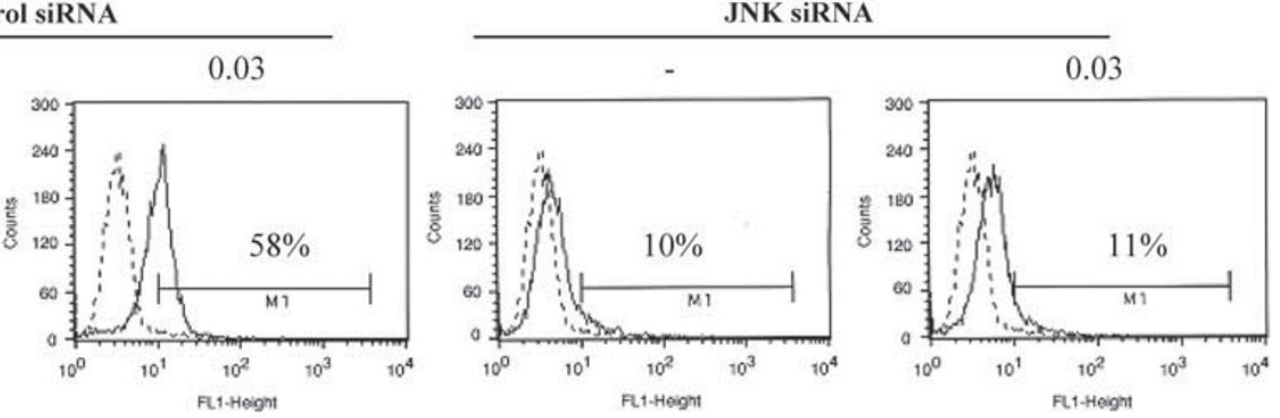

p-Aurora

IgG

p-Aurora

NB4

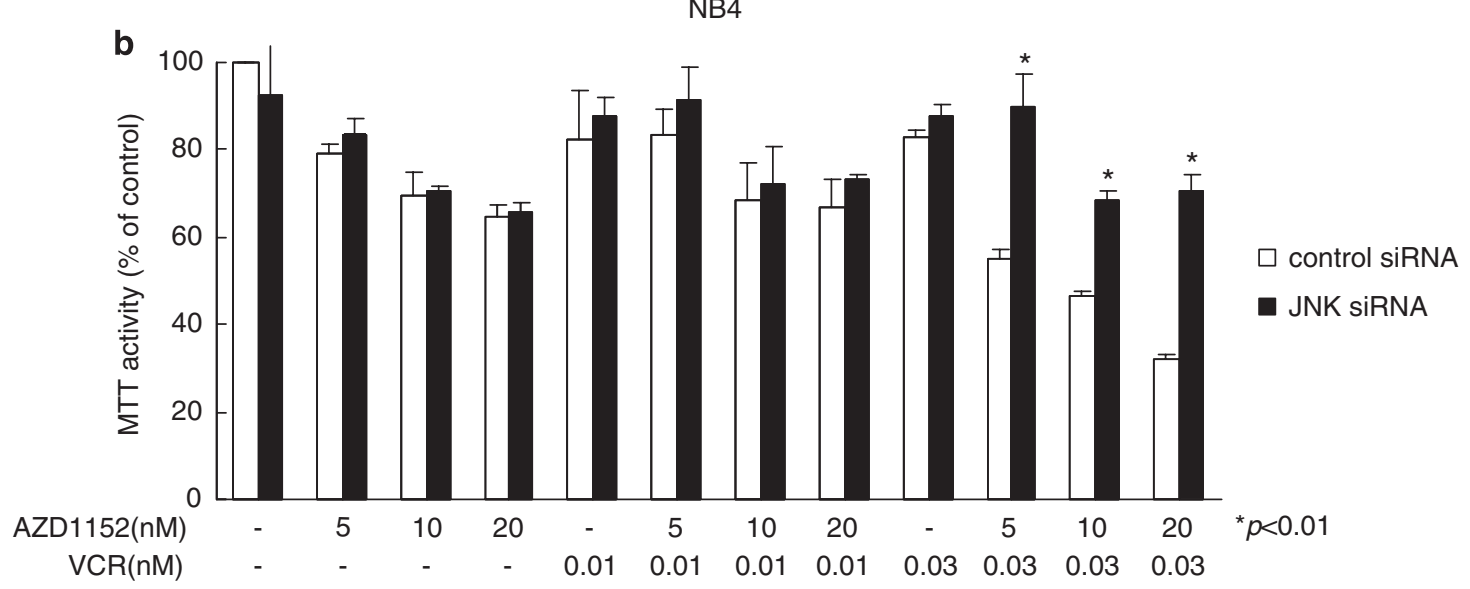

Figure 5 Downregulation of JNK by a siRNA blocked VCR-stimulated activation of Aurora kinase and attenuated synergistic growth inhibition mediated by combination of VCR and AZD1152. (a) FACS analysis. NB4 cells were transiently transfected with either control or JNK siRNA and then exposed to VCR $(0.03 \mathrm{nM})$. After $3 \mathrm{~h}$, p-JNK (upper panel) or p-Aurora kinase (lower panel) expressing population was quantified by FACScan. (b) MTT assay. NB4 cells were transiently transfected with either control or JNK siRNA, and then exposed to various concentrations of VCR and/or AZD1152. After 2 days, cell proliferation was measured by MTT assay. Results represent the mean \pm s.d. of three experiments performed in triplicate. Statistical significance of difference between control and JNK siRNA-transfected cells was determined by Student's $t$-test. FACS, fluorescence-activated cell sorting; JNK, Jun N-terminal Kinase; MTT, 3(4,5-Dimethylthiazol-2-yl)-2,5-diphenyltetrazolium bromide; VCR, vincristine.

that levels of Aurora B correlated with advanced clinical stage as well as high IPI scores (Table 1). Levels of Aurora B might be associated with a poor prognosis.
Inhibition of Aurora B kinase by the selective inhibitor AZD1152-HQPA produced profound growth arrest and apoptosis of $\mathrm{BL} /$ leukemia cells. The pan-caspase inhibitor 
z-VAD-FMK partially restored the AZD1152-HQPA-mediated growth inhibition of BL cells (Figure 2d). These observations suggest that the AZD1152-HQPA-induced growth arrest of BL cells was mediated in both a caspase-dependent and -independent manner. Further studies are required to clarify the molecular mechanisms by which blockade of Aurora B kinase induces growth inhibition and apoptosis of BL cells.

This study found that AZD1152-HQPA potentiated the ability of VCR to arrest the growth of BL cells. This finding was consistent with our previous studies, showing that AZD1152-HQPA synergized with VCR to inhibit the growth of MOLM13 AML and PALL-2 $\mathrm{Ph}^{+}$ ALL cells. ${ }^{18}$ This study explored the molecular mechanisms, which produced synergistic growth inhibition of BALM-27 cells after exposure to combination of VCR and AZD1152. Exposure of these cells to VCR increased the levels of total, as well as the phosphorylated forms of Aurora $B$ associated with the activation of JNK. Inhibition of JNK by the specific inhibitor SP600125 or the siRNA blocked VCR-stimulated phosphorylation of Aurora B, suggesting the involvement of JNK in VCR-induced activation of Aurora B kinase. Notably, when JNK was repressed by a siRNA, synergistic growth inhibition mediated by combination of VCR and AZD1152 was significantly attenuated (Figure 5). VCRstimulated Aurora B through JNK signaling may have sensitized the cells to Aurora B kinase inhibition by AZD1152HQPA. This may be the mechanism by which the combination of VCR and AZD1152-HQPA synergizes to inhibit the growth of BALM-27 cells.

Another group has shown Aurora A to be aberrantly expressed in high-grade lymphoma at the mRNA level. ${ }^{28}$ In addition, these investigators found that the downregulation of Aurora A by antisense oligonucleotides slowed the proliferation of lymphoma cells both in vitro and in vivo, ${ }^{28}$ indicating that Aurora $\mathrm{A}$ may be a promising molecular target for treatment of NHLs.

Polo-like kinase 1 (PLK1) is also recognized as a mitotic kinase and has an important role in various events in mitosis, including chromosome alignment and segregation, and cytokinesis. ${ }^{29}$ PLK1 was aberrantly expressed in DLBCLs in association with high levels of Ki-67, a marker of proliferation, and its levels were correlated with high IPI scores and poor prognosis. ${ }^{30,31}$ We have recently found that BL/leukemia cells highly expressed PLK1 and inhibition of this kinae by a specific inhibitor or a siRNA induced growth arrest and apoptosis of these cells. ${ }^{32}$ A variety of mitotic kinases may be disregulated in high-grade NHLs, and each of them can be a therapeutic target.

Both Aurora A and B kinases seem to be attractive molecular targets for the treatment of individuals with solid tumors as well as hematological malignancies. A number of small-molecule inhibitors with selectivity against Aurora A and/or Aurora B have been developed, including AZD1152 (a potent and selective inhibitor of Aurora B), MLN8054 $4^{33}$ (an orally available selective inhibitor of Aurora A), together with MK-0457, ${ }^{34}$ and PHA-739358 $8^{35}$ (a pan-Aurora kinase inhibitor). These inhibitors are undergoing evaluation in clinical trials.

Taken together, our data indicate that Aurora B kinase may be a promising molecular target for individuals with highgrade lymphoma. Combined administration of AZD1152 and other chemotherapeutic agents such as VCR, should be further investigated in the clinical setting.

\section{ACKNOWLEDGEMENTS}

We thank Dr Kirsten Mundt (AstraZeneca Pharmaceuticals, UK) for providing AZD1152-HQPA. This work was supported in part by grant-in-aid from the Ministry of Education, Culture Sports, Science and Technology of Japan (to T.I), the Kochi University President's Discretionary grant (to TI), Takeda Science Foundation (to Tl), Sagawa Foundation for Promotion of Cancer Research (to TI) and Sheryl Weissberg Lymphoma Research Foundation (to HPK). Tl contributed to the concept and design, performed the experiments, interpreted and analyzed the data, and wrote the article. $\Pi, Y A$ and MT performed the histological examination. $C N$ and JY performed the experiments. HPH provided critical revision and intellectual content. AY provided important intellectual content and gave final approval.

\section{DISCLOSURE/CONFLICT OF INTEREST}

The authors declare no conflict of interest.

1. Carmena M, Earnshaw WC. The cellular geography of aurora kinases. Nat Rev Mol Cell Biol 2003;4:842-854.

2. Marumoto T, Zhang D, Saya H. Aurora A-a guardian of poles. Nat Rev Cancer 2005;5:42-50.

3. Kimura $M$, Matsuda $Y$, Yoshioka $T$, et al. Identification and characterization of STK12/Aik2: a human gene related to aurora of Drosphila and yeast IPL1. Cytogenet Cell Genet 1998;82:147-152.

4. Tang CJ, Lin CY, Tang TK. Dynamic localization and functional implications of Aurora-C kinase during male mouse meiosis. Dev Biol 2006;290:398-410.

5. Sasai $\mathrm{K}$, Katayama $\mathrm{H}$, Stenoien $\mathrm{DL}$, et al. Aurora-C kinase is a novel chromosomal passenger protein that can complement Aurora-B kinase function in mitotic cells. Cell Motil Cytoskeleton 2004;59:249-263.

6. Bischoff JR, Anderson L, Zhu Y, et al. A homologue of Drosophila aurora kinase is oncogenic and amplified in human colorectal cancers. EMBO J 1998;17:3052-3065.

7. Zhou H, Kuang J, Zhong L, et al. Tumour amplified kinase STK15/BTAK induces centrosome amplification, aneuploidy and transformation. Nat Genet 1998;20:189-193.

8. Royce ME, Xia W, Sahin AA, et al. STK15/Aurora-A expression in primary breast tumors is correlated with nuclear grade but not with prognosis. Cancer 2004;100:12-19.

9. Sen $\mathrm{S}$, Zhou $\mathrm{H}$, White RA. A putative serine/threonine kinase encoding gene BTAK on chromosome $20 q 13$ is amplified and overexpressed in human breast cancer cell lines. Oncogene 1997;14:2195-2200.

10. Tanaka T, Kimura M, Matsunaga K. Centrosomal kinase AIK1 is overexpressed in invasive ductal carcinoma of the breast. Cancer Res 1999;59:2041-2044.

11. Chieffi P, Cozzolino L, Kisslinger A, et al. Aurora B expression directly correlates with prostate cancer malignancy and influence prostate cell proliferation. Prostate 2006;66:326-333.

12. Li D, Zhu J, Firozi PF, et al. Overexpression of oncogenic STK15/BTAK/ Aurora A kinase in human pancreatic cancer. Clin Cancer Res 2003;9:991-997.

13. Ulisse $\mathrm{S}$, Delcros JG, Baldini E, et al. Expression of Aurora kinases in human thyroid carcinoma cell lines and tissues. Int J Cancer 2006;119:275-282.

14. Reiter $R$, Gais $P$, Jutting $U$, et al. Aurora kinase a messenger RNA overexpression is correlated with tumor progression and shortened survival in head and neck squamous cell carcinoma. Clin Cancer Res 2006;12:5136-5141. 
15. Ikezoe $T$, Jing $Y$, Nishioka $C$, et al. A novel treatment strategy targeting Aurora kinases in acute myelogenous leukemia. Mol Cancer Ther 2007;6:1851-1857.

16. Ditchfield C, Johnson VL, Tighe A, et al. Aurora B couples chromosome alignment with anaphase by targeting BubR1, Mad2, and Cenp-E to kinetochores. J Cell Biol 2003;161:267-280.

17. Mortlock AA, Foote KM, Heron NM, et al. Discovery, synthesis, and in vivo activity of a new class of pyrazoloquinazolines as selective inhibitors of aurora B kinase. J Med Chem 2007;50:2213-2224.

18. Yang J, Ikezoe T, Nishioka C, et al. AZD1152, a novel and selective aurora B kinase inhibitor, induces growth arrest, apoptosis, and sensitization for tubulin depolymerizing agent or topoisomerase II inhibitor in human acute leukemia cells in vitro and in vivo. Blood 2007;110:2034-2040.

19. Wilkinson RW, Odedra R, Heaton SP, et al. AZD1152, a selective inhibitor of Aurora B kinase, inhibits human tumor xenograft growth by inducing apoptosis. Clin Cancer Res 2007;13:3682-3688.

20. Davis RJ. Signal transduction by the JNK group of MAP kinases. Cell 2000;103:239-252.

21. Ikezoe $\mathrm{T}$, Yang $\mathrm{Y}$, Taguchi $\mathrm{H}$, et al. JNK interacting protein 1 (JIP-1) protects LNCaP prostate cancer cells from growth arrest and apoptosis mediated by 12-0-tetradecanoylphorbol-13-acetate (TPA). Br J Cancer 2004;90:2017-2024.

22. Smith A, Ramos-Morales F, Ashworth A, et al. A role for JNK/SAPK in proliferation, but not apoptosis, of IL-3-dependent cells. Curr Biol 1997;7:893-896.

23. Gururajan $M$, Chui $R$, Karuppannan AK, et al. c-Jun N-terminal kinase (JNK) is required for survival and proliferation of B-lymphoma cells. Blood 2005;106:1382-1391.

24. Ikezoe T, Miller CW, Kawano S, et al. Mutational analysis of the peroxisome proliferator-activated receptor gamma gene in human malignancies. Cancer Res 2001;61:5307-5310.
25. Nishioka C, Ikezoe T, Yang J, et al. Fludarabine induces apoptosis of human T-cell leukemia virus type 1-infected T cells via inhibition of the nuclear factor-kappaB signal pathway. Leukemia 2007;21:1044-1049.

26. Ikezoe T, Yang Y, Heber D, et al. PC-SPES: a potent inhibitor of nuclear factor-kappa B rescues mice from lipopolysaccharide-induced septic shock. Mol Pharmacol 2003;64:1521-1529.

27. Chou TC, Talalay P. Quantitative analysis of dose-effect relationships: the combined effects of multiple drugs or enzyme inhibitors. Adv Enzyme Regul 1984;22:27-55.

28. Hamada M, Yakushijin $Y$, Ohtsuka $M$, et al. Aurora2/BTAK/STK15 is involved in cell cycle checkpoint and cell survival of aggressive non-Hodgkin's lymphoma. Br J Haematol 2003;121:439-447.

29. Strebhardt K, Ullrich A. Targeting polo-like kinase 1 for cancer therapy. Nat Rev Cancer 2006;6:321-330.

30. Liu L, Zhang M, Zou P. Expression of PLK1 and survivin in diffuse large B-cell lymphoma. Leuk Lymphoma 2007;48:2179-2183.

31. Mito K, Kashima K, Kikuchi $H$, et al. Expression of Polo-Like Kinase (PLK1) in non-Hodgkin's lymphomas. Leuk Lymphoma 2005;46: 225-231.

32. Ikezoe T, Yang J, Nishioka C, et al. A novel treatment strategy targeting polo-like kinase 1 in hematological malignancies. Leukemia 2009;23:1564-1576.

33. Manfredi MG, Ecsedy JA, Meetze KA, et al. Antitumor activity of MLN8054, an orally active small-molecule inhibitor of Aurora A kinase. Proc Natl Acad Sci USA 2007;104:4106-4111.

34. Harrington EA, Bebbington D, Moore J, et al. VX-680, a potent and selective small-molecule inhibitor of the Aurora kinases, suppresses tumor growth in vivo. Nat Med 2004;10:262-267.

35. Fancelli D, Moll J, Varasi $M$, et al. 1,4,5,6-Tetrahydropyrrolo[3,4c]pyrazoles: identification of a potent aurora kinase inhibitor with a favorable antitumor kinase inhibition profile. J Med Chem 2006; 49:7247-7251. 